\title{
Nonadiabatic rectification and current reversal in electron pumps
}

\author{
Alexander Croy* \\ Department of Applied Physics, Chalmers University of Technology, S-41296 Göteborg, Sweden
}

Ulf Saalmann

Max-Planck-Institute for the Physics of Complex Systems, Nöthnitzer Str. 38, D-01187 Dresden, Germany

(Received 31 January 2012; revised manuscript received 29 June 2012; published 31 July 2012)

\begin{abstract}
Pumping of electrons through nanoscale devices is one of the fascinating achievements in the field of nanoscience with a wide range of applications. To optimize the performance of pumps, operating them at high frequencies is mandatory. We consider the influence of fast periodic driving on the average charge transferred through a quantum dot. We show that it is possible to reverse the average current by sweeping the driving frequency only. In connection with this, we observe a rectification of the average current for high frequencies. Since both effects are very robust, as corroborated by analytical results for harmonic driving, they offer a new way of controlling electron pumps.
\end{abstract}

DOI: 10.1103/PhysRevB.86.035330

PACS number(s): 73.23.Hk, 05.60.Gg, 72.10.Bg, 73.63.Kv

\section{INTRODUCTION}

The possibility to pump charges or spins through nanoscale devices despite the absence of a bias voltage, ${ }^{1,2}$ shows the intriguing potential of driven nanodevices. It has consequently led to an increasing interest in such systems over the past decades. This interest was partly driven by the prospect of achieving single-electron pumping and thus creating a unique nanoscale single-electron source. However, it was soon realized that electron pumps may also help to close the metrological triangle, because they provide a connection between frequency and current. ${ }^{3,4}$ These developments were substantially aided by the rapid experimental and technological progress in controlling and fabricating nanoscale devices. In particular, it recently became possible to realize charge pumping in the $\mathrm{GHz}$ regime ${ }^{5-8}$ which leads to a significant increase of the pumped current.

The adiabatic limit of charge pumping, where the driving frequency $\Omega$ is much smaller than the typical charging/ discharging rate $\Gamma$, is very well understood. ${ }^{9}$ In this case, one can resort to well-established time-independent formalisms to describe the electron transport. ${ }^{9-13}$ The opposite limit of very fast pumping has also been studied ${ }^{14,15}$ to a large extent in the context of photon-assisted tunneling. ${ }^{16-18}$ However, the borderland between these limits is lacking a comparably systematic understanding. Here, one is faced with an inherently nonequilibrium problem, while due to the similar timescales a perturbative description is not possible. To address this problem, numerical calculations in the time-domain ${ }^{19,20}$ or methods based on Floquet theory have typically been used. $^{21-25}$ Only recently, within the diagrammatic real-time transport theory, has a summation to all orders in $\Omega$ in the limit of weak tunnel-coupling and moderate pumping frequencies been achieved, ${ }^{13}$ revealing interesting nonadiabatic effects for spin and charge pumping. Additionally, in particular for low temperatures, electron-electron interactions become important and can influence the pumping behavior. ${ }^{26}$

In this article we demonstrate the drastic consequences of nonadiabatic driving for the pumped charge per period by showing that it is possible to reverse the average current just by sweeping the driving frequency. Such current reversals have been seen in multilevel systems, ${ }^{22,23}$ where they are due resonance effects and as such are sensitive to the respective couplings and energies. Here the reversal is observed for a simple and generic system. Since it does not rely on interference effects and or any particular driving scheme, the effect is very robust and thus likely to be realized experimentally. Moreover, the underlying mechanisms of both the reversal effect and the associated effect of nonadiabatic rectification of the pumped current for high frequencies, can be understood in full detail.

\section{MODEL AND METHODS}

The electron pump is realized using a quantum dot (QD), which is coupled via tunnel barriers to a left (L) and a right $(\mathrm{R})$ contact. These are connected to larger electron reservoirs. The total Hamiltonian is $H=H_{\text {dot }}+H_{\text {res }}+$ $H_{\text {tun }}$, where the first term describes the QD itself, $H_{\mathrm{dot}}=$ $\varepsilon(t) \hat{c}^{\dagger} \hat{c}$, the second term characterizes the attached contacts, $H_{\text {res }}=\sum_{\alpha \in \mathrm{L}, \mathrm{R}} \sum_{k s} \varepsilon_{\alpha k} \hat{b}_{\alpha k}^{\dagger} \hat{b}_{\alpha k}$, and the third term accounts for the tunnel coupling between QD and contacts, $H_{\text {tun }}=$ $\sum_{\alpha k} T_{\alpha k}(t) \hat{b}_{\alpha k}^{\dagger} \hat{c}+$ H.c. Here, $\hat{c}^{\dagger}$ and $\hat{b}_{\alpha k}^{\dagger}$ create an electron in the QD state and in the reservoir state $\alpha k$, respectively. The transport through the QD is mainly characterized by the tunneling rate $\Gamma_{\alpha}(t)=2 \pi \sum_{k}\left|T_{\alpha k}(t)\right|^{2} \delta\left(\varepsilon-\varepsilon_{\alpha k}\right)$, which is given in terms of the time-dependent tunneling amplitudes $T_{\alpha k}(t)$. In order to calculate the pumped charge per period, we use the framework of nonequilibrium Green functions (NEGFs) ${ }^{27,28}$ in conjunction with an auxiliary mode expansion. ${ }^{29}$ This method is very flexible and allows for treating arbitrary time-dependencies of the parameters entering the Hamiltonian.

Specifically, we use the following time dependence for the QD level and the couplings to the reservoirs:

$$
\begin{aligned}
\varepsilon(t) & =\varepsilon_{0}+\varepsilon_{1} \cos (\Omega t), \\
\Gamma_{\alpha}(t) & =\Gamma_{\alpha}^{0} \exp \left[\eta_{\alpha} \cos \left(\Omega t-\delta_{\alpha}\right)\right] .
\end{aligned}
$$

This choice reflects the experimental situation of modulated voltages, which will in general lead to an exponential dependence of the tunnel couplings on this modulation. ${ }^{7,8}$ The 


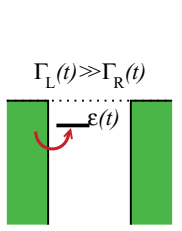

charging

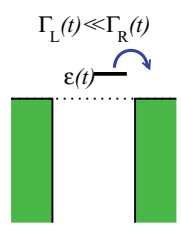

discharging

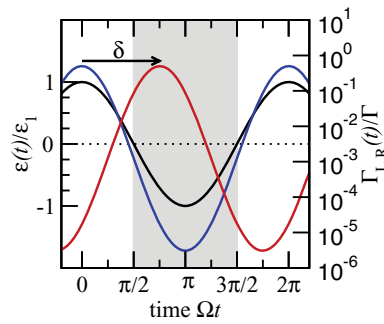

FIG. 1. (Color online) Schematic representation of the pumping device: The level $\varepsilon$ and the couplings $\Gamma_{\mathrm{L}, \mathrm{R}}$ to left and right reservoir oscillate in time, inducing periodic charging and discharging. Time dependence of $\varepsilon$ (black line, left axis) and $\Gamma_{\mathrm{L}, \mathrm{R}}$ (red and blue lines, right axis) according to Eqs. (1) for a phase delay of $\delta=3 \pi / 4$.

dimensionless parameter $\eta$ quantifies the dynamic range of the couplings within one cycle. The value $\eta=6$ used below, is motivated by estimates for experimental electron pumps. ${ }^{7}$ The most important aspect of Eq. (1b) is, however, the inclusion of the phase shifts $\delta_{\alpha}$ allowing for an offset of the oscillations with respect to each other. For $\delta_{\mathrm{L}}=-\delta_{\mathrm{R}}= \pm \pi / 2$ the left and the right coupling are maximally out-of-phase when the energy level crosses the Fermi levels of the reservoirs, which yields an optimal turnstile operation. ${ }^{30}$ In the following, as in earlier work, ${ }^{7}$ we use $\delta_{\mathrm{L}} \equiv \delta$ and $\delta_{\mathrm{R}}=0$. This corresponds to a single-gate modulation setup. In Fig. 1 the time dependence of $\varepsilon$ and $\Gamma_{\alpha}$ is shown for a specific delay of $\delta=3 \pi / 4$.

The time-dependent driving (1) induces currents $J_{\mathrm{L}}$ and $J_{\mathrm{R}}$ from the left and the right reservoir, respectively. The net charge, pumped from the left to the right reservoir within one period $\tau \equiv 2 \pi / \Omega$, can be obtained by the integral

$$
Q=\frac{1}{2} \int_{0}^{\tau} d t^{\prime}\left[J_{\mathrm{L}}\left(t^{\prime}\right)-J_{\mathrm{R}}\left(t^{\prime}\right)\right] .
$$

In numerical calculations, the respective equations are propagated until the charge per period converges.

Additionally, we analyze the pumping using a simple rateequation description of the electron transport. As we will show, this description is sufficient to reveal the basic mechanisms behind both effects. The currents $J_{\mathrm{L}, \mathrm{R}}$ and the dot occupation $n$ are given by the following equations ${ }^{7}$ :

$$
\begin{aligned}
J_{\mathrm{L}, \mathrm{R}}(t) & =\Gamma_{\mathrm{L}, \mathrm{R}}(t)[f(\varepsilon(t))-n(t)], \\
\partial_{t} n(t) & =J_{\mathrm{L}}(t)+J_{\mathrm{R}}(t),
\end{aligned}
$$

with $f(\varepsilon)$ the Fermi distribution function describing the occupation in the reservoirs.

The rate-equation approach is valid for weak couplings $\Gamma_{\mathrm{L}, \mathrm{R}} \ll k_{\mathrm{B}} T$ and large oscillation amplitudes $\varepsilon_{1} \gg k_{\mathrm{B}} T$ as has been discussed (and termed semiclassical approximation) in a different context before. ${ }^{31}$ Note that in our case the couplings $\Gamma_{\mathrm{L}, \mathrm{R}}$ and the quantum-dot level $\varepsilon$ oscillate with the same frequency.

\section{RESULTS AND DISCUSSION}

Figure 2 shows the numerical NEGF results for the pumped charge $Q$ as a function of frequency $\Omega$ and phase shift $\delta$. For very low frequencies, $\Omega \ll \Gamma$, one finds the expected behavior of $Q$ in dependence on $\delta$ : For negative shifts the pumped charge is negative, while for positive shifts it is positive,

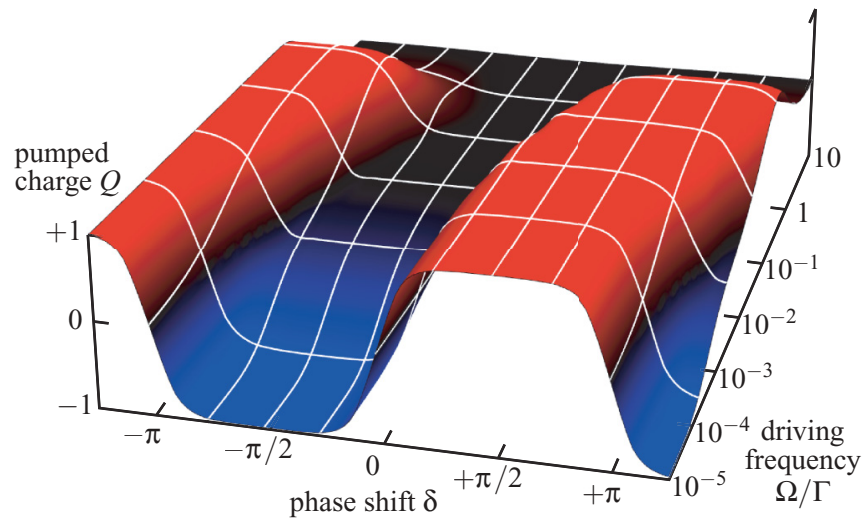

FIG. 2. (Color online) Pumped charge $Q$ per period according to Eq. (2) as a function of frequency $\Omega$ and phase shift $\delta$ obtained from an NEGF calculation. The parameters defining the driving and the reservoirs are as follows: $\varepsilon_{0}=0, \varepsilon_{1}=20 \Gamma, \eta_{\mathrm{L}, \mathrm{R}}=6, \Gamma_{\mathrm{L}, \mathrm{R}}^{0}=$ $\Gamma e^{-\eta_{\alpha}} / 2, \mu_{\mathrm{L}, \mathrm{R}}=0$, and $k_{\mathrm{B}} T=\Gamma / 10$.

which is known as peristaltic pumping. ${ }^{9}$ In striking contrast, one observes for higher frequencies $\left(\Omega \gtrsim 10^{-2} \Gamma\right)$ that the net current always flows in one direction. This implies for a negative phase delay $(\delta \approx-3 \pi / 4)$ that by sweeping the driving frequency one can change the sign of the pumped current per period or, in other words, reverse the direction of the average current. These effects-rectification and current reversal-are the central result of this article.

We have verified numerically that the pumped charge obtained within the rate-equation model (3) agrees very well with the NEGF result as can be seen in Fig. 3, which shows the current reversal in panel a ( $Q$ vs frequency for $\delta=-3 \pi / 4)$ and the rectification in panel b ( $Q$ vs phase shift for $\Omega=\Gamma / 10$ ). Thus we can analyze the time evolution by means of the simple rate equations (3).

\section{A. Fourier analysis}

In order to get a better understanding of this surprising behavior, it is instructive to examine the temporal evolution of the currents for slow and fast drivings, respectively. This is
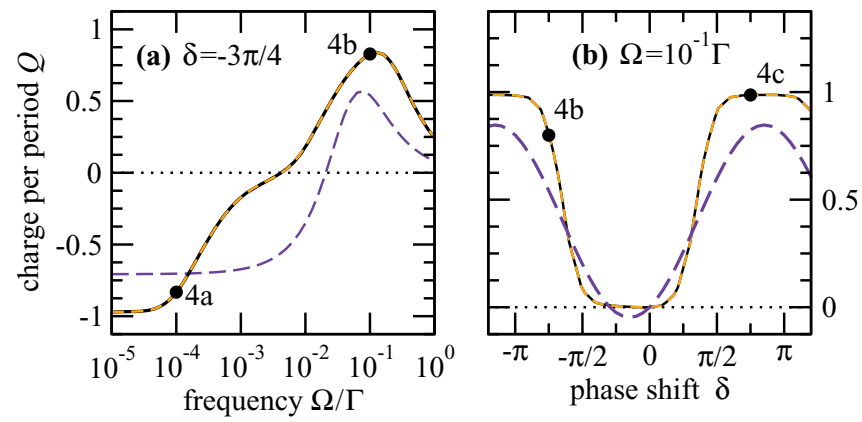

FIG. 3. (Color online) Pumped charge per period $Q$ for the same parameters as in Fig. 2. NEGF (black lines) and rate-equation (broken orange) results are indistinguishable. a) Current reversal for the phase shift $\delta=-3 \pi / 4$. b) Current rectification for the driving frequency $\Omega=\Gamma / 10$. Parameters for which the time evolution is shown in Fig. 4 are marked by black points. Result for the harmonic model [Eq. (12) with $\left.\Gamma_{0}=\Gamma_{1}=\Gamma / 20\right]$ are shown as indigo/long-dashed line. 
most conveniently done using a Fourier analysis. By means of the definition (2) and the equation of motion (3) the pumped charge per period reads

$$
Q=\frac{1}{2} \int_{0}^{\tau} d t^{\prime} \Gamma^{(-)}\left(t^{\prime}\right)\left[f\left(\varepsilon\left(t^{\prime}\right)\right)-n\left(t^{\prime}\right)\right] .
$$

It is given in terms of the difference of the tunnel couplings $\Gamma^{(-)}$, which is defined (along with the corresponding sum $\Gamma^{(+)}$ which will be used later) as follows:

$$
\Gamma^{( \pm)}(t) \equiv \Gamma_{\mathrm{L}}(t) \pm \Gamma_{\mathrm{R}}(t)=\sum_{m} \Gamma_{m}^{( \pm)} e^{\mathrm{i} m \Omega t}
$$

Here, $\Gamma_{m}^{( \pm)}=\Gamma_{\mathrm{L}}^{0} I_{m}\left(\eta_{\mathrm{L}}\right) e^{\mathrm{i} m \delta \pi} \pm \Gamma_{\mathrm{R}}^{0} I_{m}\left(\eta_{\mathrm{R}}\right)$ with $I_{m}$ being the modified Bessel function of the first kind of order $m$. The last expression is the Fourier series of the tunnelingrate sum/difference. Analogously, the occupation $n(t)=$ $\sum_{m} n_{m} e^{\mathrm{i} m \Omega t}$ and the Fermi function of the time-dependent level $f(\varepsilon(t))=\sum_{m} f_{m} e^{\mathrm{i} m \Omega t}$ can be expanded in a Fourier series. For sufficiently low temperatures, $k_{\mathrm{B}} T \ll \varepsilon_{1}$, we can replace the Fermi function by the step function $f(\varepsilon(t))=\Theta(-$ $\varepsilon(t))$ and get $f_{m} \equiv \Theta_{m}$ with $\Theta_{0}=1 / 2, \Theta_{m}=(-1)^{(m+1) / 2} / m \pi$ for odd $m$, and $\Theta_{m}=0$ for even $m \neq 0$.

Plugging these series into Eqs. (3) yields an algebraic equation for the Fourier coefficients of the occupation, which reads in matrix-vector notation

$$
\mathrm{i} \Omega \mathbb{D} \cdot \boldsymbol{n}=\mathbb{G} \cdot[\boldsymbol{\Theta}-\boldsymbol{n}],
$$

with the time-derivative operator $D_{k m} \equiv k \delta_{m k}$ and the coupling matrix $G_{k m} \equiv \Gamma_{k-m}^{(+)}$. The components of the vectors $\boldsymbol{\Theta}$ and $\boldsymbol{n}$ are given by the Fourier coefficients introduced above. Additionally, for notational convenience, we will use the vector $\overline{\boldsymbol{\Gamma}}^{( \pm)}$whose components are reversed, $\bar{\Gamma}_{m}^{( \pm)} \equiv \Gamma_{-m}^{( \pm)}$. It is important to notice, that neither $\mathbb{D}$ nor $\mathbb{G}$ contain the frequency $\Omega$. This allows for a straightforward expansion in powers of $\Omega$, as we will show below. For the charge $Q$ in Eq. (4) one needs $\boldsymbol{\Theta}-\boldsymbol{n}$, which can be easily obtained from Eq. (6) yielding

$$
\begin{aligned}
Q & =\frac{\pi}{\Omega} \overline{\boldsymbol{\Gamma}}^{(-)} \cdot[\boldsymbol{\Theta}-\boldsymbol{n}], \\
\boldsymbol{\Theta}-\boldsymbol{n} & =\left[\mathbb{D}-\frac{\mathrm{i}}{\Omega} \mathbb{G}\right]^{-1} \mathbb{D} \boldsymbol{\Theta} .
\end{aligned}
$$

Note that this expression only depends on given quantities, which are either external parameters (like $\overline{\boldsymbol{\Gamma}}^{(-)}$or $\Omega$ ) or trivial matrices (like $\mathbb{D}$ ). With this formulation one can derive intuitive expressions for low- and high-frequency pumping. In order to invert the matrix in Eq. (7b) we split the matrix $\mathbb{G} / \Omega=$ $\mathbb{G}_{0}+\mathbb{G}_{1}$ into a diagonal and an off-diagonal component

$$
\begin{aligned}
& \left(G_{0}\right)_{k m} \equiv \delta_{k m} G_{k m} / \Omega=\delta_{k m} \Gamma_{0} / \Omega, \\
& \left(G_{1}\right)_{k m} \equiv\left(1-\delta_{k m}\right) G_{k m} / \Omega,
\end{aligned}
$$

where we have used in Eq. (8a) that all elements of the diagonal $G_{k k}=I_{0}\left(\eta_{\mathrm{L}}\right)+I_{0}\left(\eta_{\mathrm{R}}\right) \equiv \Gamma_{0}$. With the two contributions in Eqs. (8) one can rewrite Eq. (7b) in terms of the expansion

$$
\begin{aligned}
\boldsymbol{\Theta}-\boldsymbol{n} & =\sum_{k=0}^{\infty} \mathbb{K}_{k} \mathbb{D} \boldsymbol{\Theta} \\
\mathbb{K}_{k} & \equiv\left(\mathbb{D}-\mathrm{i} \mathbb{G}_{0}\right)^{-1}\left[\mathbb{G}_{1}\left(\mathbb{D}-\mathrm{i} \mathbb{G}_{0}\right)^{-1}\right]^{k},
\end{aligned}
$$

where $\mathbb{D}-\mathbf{i} \mathbb{G}_{0}$ can be easily inverted since it is diagonal. Equations (9) have a very intuitive interpretation. The Fourier vector $\mathbb{D} \Theta$ describes alternating " $\delta$-kicks" at times $\Omega t_{j}=(j+1 / 2) \pi$. The expansion in terms of $\mathbb{K}_{k}$ accounts for the response of the system to these kicks, which is mainly characterized by the ratio $\Gamma_{0} / \Omega$. The first term $\mathbb{K}_{0}=\left(\mathbb{D}-\mathrm{i} \mathbb{G}_{0}\right)^{-1}$ in the sum is diagonal and given as

$$
\left(K_{0}\right)_{m m}=\frac{m+\mathrm{i} \Gamma_{0} / \Omega}{m^{2}+\left(\Gamma_{0} / \Omega\right)^{2}} .
$$

The Lorentzian decay in the index $m$ accounts for the exponential charging or discharging of the quantum dot. It is interesting to consider the following limits:

$$
\begin{aligned}
& \Gamma_{0} / \Omega \gg 1: \mathbb{K}_{0}=\mathrm{i} \frac{\Omega}{\Gamma_{0}} \mathbb{I}+\left(\frac{\Omega}{\Gamma_{0}}\right)^{2} \mathbb{D}+\cdots, \\
& \Gamma_{0} / \Omega \ll 1: \mathbb{K}_{0}=\tilde{\mathbb{D}}^{-1}+\mathrm{i} \frac{\Gamma_{0}}{\Omega} \tilde{\mathbb{D}}^{-2}+\cdots
\end{aligned}
$$

with $\tilde{D}_{k k} \equiv D_{k k}$ for $k \neq 0$ and $\tilde{D}_{00} \equiv-\mathrm{i} \Gamma_{0} / \Omega$, which replaces $\mathbb{D}$ to enable the matrix inversion.

\section{B. Time evolution for slow and fast driving}

In order to investigate the different limits in Eq. (11) we show in Fig. 4 the time evolution within one period of the occupation $n(t)$ and the transferred charge (upper row), as well as the currents $J_{\mathrm{L}}(t)$ and $J_{\mathrm{R}}(t)$ (lower row) obtained from Eqs. (3). Note that the figure shows the time evolution in units of $\Omega^{-1}$, which is $10^{3}$ times larger in Fig. 4(a) compared to Figs. 4(b) and 4(c), respectively. In absolute times the intervals when current flows are similar for $\Omega=10^{-4}$ and $\Omega=10^{-1}$. However, in units of $\Omega^{-1}$ the interval for the lower frequencies is much shorter than the driving period $\tau$.

In the adiabatic limit $(\Omega / \Gamma \rightarrow 0)$ given by Eq. (11a), all matrix elements of $\mathbb{K}_{0}$ are identical. This implies that $\boldsymbol{\Theta}-\boldsymbol{n} \propto$ $\mathbb{D} \Theta$, i.e., the " $\delta$-kicks" of the driving mentioned before also occur in the response of the system. In other words, the electron in- or outflow is much faster than the external period. Indeed this can be seen in the lower panel of Fig. 4(a), which shows that the current flows mainly at the instants of time $t_{j}$ when the level energy crosses the Fermi level of the reservoirs. Therefore, $Q$ in Eq. (4) is determined by the couplings at those specific times $\Gamma^{(-)}\left(t_{j}\right)$. For negative phase shifts $-\pi<\delta<0$, as shown in Fig. 4(a), it is $\Gamma^{(-)}<0$ for the charging at $\Omega t_{0}=\pi / 2$ and $\Gamma^{(-)}>0$ for the discharging at $\Omega t_{1}=3 \pi / 2$. The opposite applies for positive shifts $0<\delta<+\pi$. As mentioned before, the pumping is "peristaltic". 9 The simple relation of $Q$ and the coupling differences $\Gamma^{(-)}\left(t_{0}\right)$ and $\Gamma^{(-)}\left(t_{1}\right)$ explains that the maximal charge $Q$ is obtained for $\delta= \pm \pi / 2$ and that it vanishes for $\delta=0, \pm \pi$. Because of the prefactor in Eq. (4), $Q$ becomes independent of $\Omega$ in the adiabatic case and the first nonadiabatic correction is proportional to $\Omega$. 


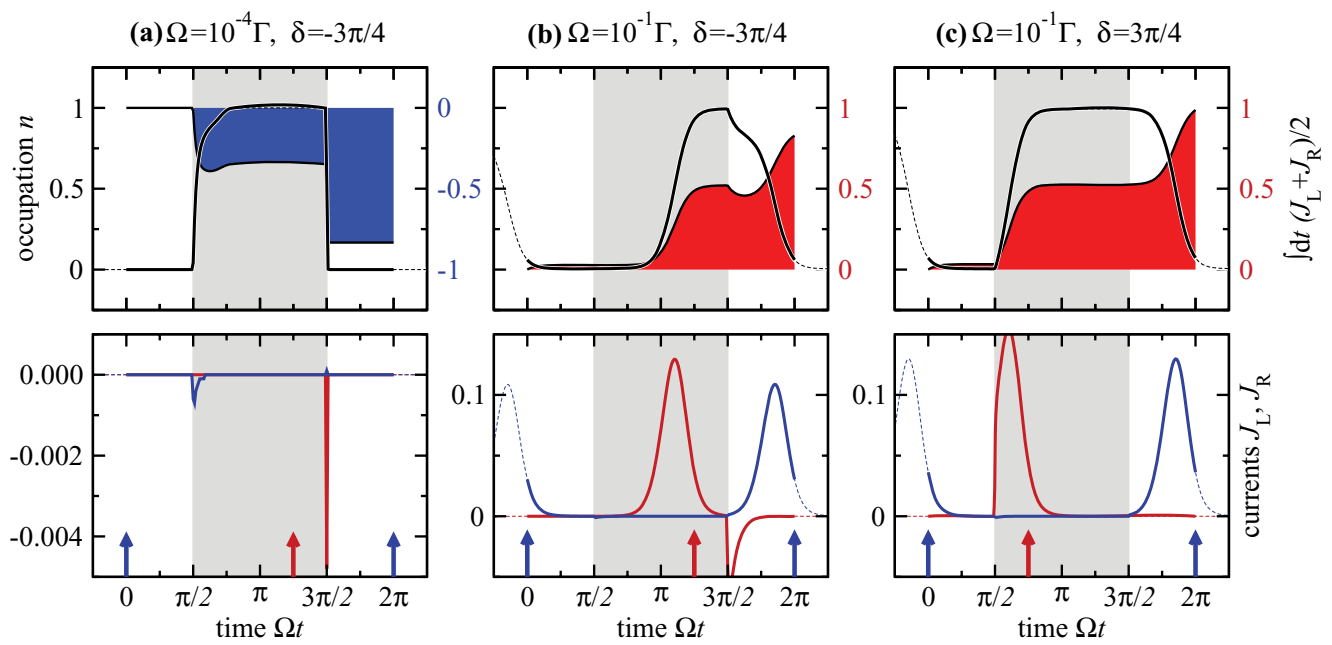

FIG. 4. (Color online) Time evolution within one period $\tau=2 \pi / \Omega$ for three different parameters sets $(\Omega, \delta)$. Note that the time is given in units of $\Omega^{-1}$, which implies that absolute times in column a) are by a factor of $10^{3}$ larger than in columns b) and c). Lower row: Currents through tunnel barriers $J_{\mathrm{L}}(t)$ and $J_{\mathrm{R}}(t)$ (red and blue lines). Upper row: Dot occupation $n(t)$ with its scale on the left side and transferred charge $\int^{t} d t^{\prime}\left(J_{\mathrm{L}}\left(t^{\prime}\right)-J_{\mathrm{R}}\left(t^{\prime}\right)\right) / 2$ with its scale on the right side. The gray area indicates the time span when the dot is charged, i.e., $\varepsilon(t)<0$. Red/blue arrows mark the times when left/right couplings are maximal.

On the other hand, in the fast driving limit $(\Omega / \Gamma \rightarrow \infty)$ given by Eq. (11b), we get $\boldsymbol{\Theta}-\boldsymbol{n} \propto \boldsymbol{\Theta}+$ const and the occupation $n(t)$ is constant. Because of Eq. (4) there is no transfer in this limit and $Q \propto \Omega^{-1}$. In between these two extrema the time scale of the exponential decay is comparable to the period $\tau$ of the external driving. Thus the integral in Eq. (4) "considers" $\Gamma^{(-)}(t)$ over the whole period, not just at particular instants of time as in the adiabatic case. In the lower panels of Figs. 4(b) and 4(c) the resulting time-dependence of the currents can be seen for $\delta= \pm 3 \pi / 4$ and $\Omega=\Gamma / 10$. This explains why the net current flows in the same direction as long as the peak of the left coupling occurs during charging of the dot, which is fulfilled for $|\delta|>\pi / 2$. This time-interval is indicated by the gray areas in Fig. 4. For $\delta=-3 \pi / 4$ (or equivalently $\delta=+5 \pi / 4$, cf. Fig. 4(b)) the charging occurs in the second half of the charging period, for $\delta=+3 \pi / 4$ [Fig. 4(c)] in the first half. Thus, in both cases the dot is charged from the left and discharged to right since the right coupling $\Gamma_{\mathrm{R}}$ is locked to the oscillating level $\varepsilon$. This explains the observed rectification effect.

By means of this interpretation one expects a current reversal for phase delays $-\pi<\delta<-\pi / 2$. For these delays it is $\Gamma^{(-)}\left(t_{0}\right)<0$, relevant for small $\Omega$, but $\int_{t_{0}}^{t_{1}} \mathrm{~d} t^{\prime} \Gamma^{(-)}\left(t^{\prime}\right)>0$, relevant for large $\Omega$, and the charging occurs either form the right or the left. Correspondingly, it is $\Gamma^{(-)}\left(t_{1}\right)>0$ and $\int_{t_{1}}^{t_{2}} d t^{\prime} \Gamma^{(-)}\left(t^{\prime}\right)<0$ and the dot is discharged to the opposite direction. Figure 2 indeed shows this behavior in the predicted range of phase delays $\delta$.

Thus we conclude that the reversal occurs when the current changes its behavior from being dominated by the couplings at the instants of time at which the oscillating level $\varepsilon$ crosses the Fermi level of the contacts (adiabatic limit) to being defined by the couplings during the periods of time in which $\varepsilon$ is below or above the Fermi level (nonadiabatic limit). In order to estimate the reversal frequency $\Omega^{*}$ at which this transition occurs, we compare $\Omega$ with the couplings $\Gamma_{\mathrm{L}, \mathrm{R}}$ at the crossing times $\Omega t_{0}=\pi / 2$ and $\Omega t_{1}=3 \pi / 2$. If $\Omega$ is significantly smaller than these couplings the behavior is adiabatic, since the dot is loaded or unloaded in a small fraction of the driving period $\tau$. In the opposite limit the behavior is nonadiabatic. Notice that this crossover may occur at different frequencies for charging and decharging.

Due to the dependence of $\Gamma_{\mathrm{L}}$ on the delay $\delta$, the reversal frequency $\Omega^{*}$ also depends on $\delta$. We will estimate its value for $\delta=-3 \pi / 4$, the case shown in Fig. 3(a). According to Eq. (1b) the relevant couplings are $\Gamma_{\mathrm{R}}\left(t_{0}\right)=(\Gamma / 2) \exp (-\eta) \approx$ $10^{-2.9} \Gamma$ and $\Gamma_{\mathrm{L}}\left(t_{1}\right)=(\Gamma / 2) \exp ((1 / \sqrt{2}-1) \eta) \approx 10^{-1.1} \Gamma$ with $\Gamma_{\mathrm{L}}\left(t_{0}\right) \ll \Gamma_{\mathrm{R}}\left(t_{0}\right)$ and $\Gamma_{\mathrm{R}}\left(t_{1}\right) \ll \Gamma_{\mathrm{L}}\left(t_{1}\right)$. Indeed we find different reversal frequencies and use as an upper estimate $\Omega^{*} \lesssim$ $\left[\Gamma_{\mathrm{R}}\left(t_{0}\right) \Gamma_{\mathrm{L}}\left(t_{1}\right)\right]^{1 / 2}$. For the parameters used in the numerical calculations one obtains $\Omega^{*} \lesssim 10^{-2} \Gamma$, which is a good estimate for the numerical value $\Omega^{*} \approx 10^{-2.4} \Gamma$ seen in Fig. 3(a).

We would like to stress that the apparent reversal frequency $\Omega^{*}$ and the frequency range over which the pumped current is reversed depend on the time dependence of the couplings, in particular on the dynamic-range parameter $\eta$. For smaller $\eta$ the reversal frequency decreases and the frequency range shrinks.

\section{Harmonic driving}

To show that the current reversal is not specific to our driving scheme, we turn to the case of purely harmonic driving, i.e., $\Gamma(t)=\Gamma_{0}+\Gamma_{1} \cos (\Omega t-\delta)$. More importantly, the basic mechanism of the current reversal can be understood analytically in this case. Harmonic driving at a frequency $\Omega$ is characterized by having three Fourier components $\Gamma_{\mathrm{L}}=$ $\left\{\Gamma_{1} \mathrm{e}^{+\mathrm{i} \delta}, \Gamma_{0}, \Gamma_{1} \mathrm{e}^{-\mathrm{i} \delta}\right\}$ and $\boldsymbol{\Gamma}_{\mathrm{R}}=\left\{\Gamma_{1}, \Gamma_{0}, \Gamma_{1}\right\}$, where $\Gamma_{0}>\Gamma_{1}$ guarantees positive couplings and $\delta$ is the time shift of the left and right coupling. If the calculation in Eq. (9a) is restricted to $\mathbb{K}_{0}$, only three Fourier components of the step function are needed, which are $\Theta=\{-1 / \pi, 1 / 2,-1 / \pi\}$. Using these 
expressions in Eqs. (7)-(10) one gets

$$
Q=\frac{\Gamma_{1}}{\Gamma_{0}^{2}+\Omega^{2}}\left[\Omega(1-\cos \delta)+\Gamma_{0} \sin \delta\right] .
$$

This simple expression contains all the basic features for periodic pumping including the current reversal. Moreover, it allows us to analyze the respective regimes in detail.

For $\Omega \ll \Gamma_{0}$ (adiabatic limit) one obtains from Eq. (12) $Q=\left(\Gamma_{1} / \Gamma_{0}\right) \sin \delta$. As discussed earlier, the sign of $Q$ depends on the order of the "door openings". Optimal transfer is attained for $\Gamma_{1}=\Gamma_{0}$. In the opposite limit, $\Omega \gg \Gamma_{0}$, one finds $Q=\left(\Gamma_{1} / \Omega\right)(1-\cos \delta)$. Most interestingly and in contrast to the adiabatic case, in this limit the sign of $Q$ is independent of the phase shift $\delta$, which is the nonadiabatic rectification effect. Consequently, for $\delta<0$ one gets negative $Q$ in the adiabatic and positive $Q$ in the nonadiabatic limit: the average current can be reversed by tuning the driving frequency. These findings are confirmed by Fig. 3, where we compare the harmonic driving to the scenario considered initially [Eqs. (1)]. Qualitatively, the behavior of $Q$ is quite similar in both cases, which underlines the robustness of the discussed effects.

Finally, the simple expression (12) is also in qualitative agreement with the time-dependent behavior for the driving scheme discussed in detail in Sec. III B. A current reversal is expected for $-\pi<\delta<-\pi / 2$ since for these delays $1-\cos \delta>1$ and $\sin \delta<0$. According to Eq. (12) the reversal occurs for $\delta=-3 \pi / 4$ at the frequency
$\Omega^{*}=(\sqrt{2}-1) \Gamma_{0} \approx 10^{-1.7} \Gamma$, which is in good agreement with the result shown in Fig. 3(a).

\section{SUMMARY}

In summary, we have studied the influence of non-adiabatic driving on the charge pumping through a quantum dot in the Coulomb-blockade regime. Our numerical calculations, based on a NEGF method, showed that the average pumped current can be reversed by sweeping the driving frequency. The origin of this effect was found to be the qualitatively different response to slow and fast driving, rendering the difference of the left and right tunneling rates matter only at specific instants of time (adiabatic case) or during a time-interval (nonadiabatic case). By means of a description with rate equations, we derived for the case of harmonic driving an analytical expression for the transferred charge per cycle, which confirms our analysis. Furthermore this shows that the observed effects are generic and quite robust with respect to the specific form of the external driving. Therefore they could be useful for realizing frequency filters or frequency-selected switches.

\section{ACKNOWLEDGMENTS}

We thank Zach Walters for proofreading an earlier version of this manuscript. *croy@chalmers.se

${ }^{1}$ D. J. Thouless, Phys. Rev. B 27, 6083 (1983).

${ }^{2}$ L. P. Kouwenhoven, A. T. Johnson, N. C. van der Vaart, C. J. P. M. Harmans, and C. T. Foxon, Phys. Rev. Lett. 67, 1626 (1991).

${ }^{3}$ J. Flowers, Science 306, 1324 (2004).

${ }^{4}$ M. W. Keller, Metrologia 45, 102 (2008).

${ }^{5}$ M. D. Blumenthal et al., Nat. Phys. 3, 343 (2007).

${ }^{6}$ A. Fujiwara, K. Nishiguchi, and Y. Ono, Appl. Phys. Lett. 92, 042102 (2008).

${ }^{7}$ B. Kaestner et al., Phys. Rev. B 77, 153301 (2008).

${ }^{8}$ S. P. Giblin et al., New J. Phys. 12, 073013 (2010).

${ }^{9}$ P. W. Brouwer, Phys. Rev. B 58, R10135 (1998).

${ }^{10}$ F. Zhou, B. Spivak, and B. Altshuler, Phys. Rev. Lett. 82, 608 (1999).

${ }^{11}$ O. Entin-Wohlman, A. Aharony, and Y. Levinson, Phys. Rev. B 65, 195411 (2002).

${ }^{12}$ M. Moskalets and M. Büttiker, Phys. Rev. B 64, 201305(R) (2001).

${ }^{13}$ F. Cavaliere, M. Governale, and J. König, Phys. Rev. Lett. 103, 136801 (2009).

${ }^{14}$ B. L. Hazelzet, M. R. Wegewijs, T. H. Stoof, and Y. V. Nazarov, Phys. Rev. B 63, 165313 (2001).

${ }^{15}$ M. Braun and G. Burkard, Phys. Rev. Lett. 101, 036802 (2008).

${ }^{16}$ C. Bruder and H. Schoeller, Phys. Rev. Lett. 72, 1076 (1994).
${ }^{17}$ R. H. Blick, R. J. Haug, D. W. van der Weide, K. von Klitzing, and K. Eberl, Appl. Phys. Lett. 67, 3924 (1995).

${ }^{18}$ T. H. Oosterkamp, L. P. Kouwenhoven, A. E. A. Koolen, N. C. van der Vaart, and C. J. P. M. Harmans, Phys. Rev. Lett. 78, 1536 (1997).

${ }^{19}$ V. Moldoveanu, V. Gudmundsson, and A. Manolescu, Phys. Rev. B 76, 165308 (2007).

${ }^{20}$ G. Stefanucci, S. Kurth, A. Rubio, and E. K. U. Gross, Phys. Rev. B 77, 075339 (2008).

${ }^{21}$ C. A. Stafford and N. S. Wingreen, Phys. Rev. Lett. 76, 1916 (1996).

${ }^{22}$ M. Moskalets and M. Büttiker, Phys. Rev. B 66, 205320 (2002).

${ }^{23}$ J. Lehmann, S. Kohler, P. Hänggi, and A. Nitzan, Phys. Rev. Lett. 88, 228305 (2002).

${ }^{24}$ M. Strass, P. Hänggi, and S. Kohler, Phys. Rev. Lett. 95, 130601 (2005).

${ }^{25}$ L. Arrachea and M. Moskalets, Phys. Rev. B 74, 245322 (2006).

${ }^{26}$ L. Arrachea, A. Levy Yeyati, and A. Martin-Rodero, Phys. Rev. B 77, 165326 (2008).

${ }^{27}$ N. S. Wingreen, A.-P. Jauho, and Y. Meir, Phys. Rev. B 48, 8487 (1993).

${ }^{28}$ A.-P. Jauho, N. S. Wingreen, and Y. Meir, Phys. Rev. B 50, 5528 (1994).

${ }^{29}$ A. Croy and U. Saalmann, Phys. Rev. B 80, 245311 (2009).

${ }^{30}$ F. Battista and P. Samuelsson, Phys. Rev. B 83, 125324 (2011).

${ }^{31}$ D. C. Langreth and P. Nordlander, Phys. Rev. B 43, 2541 (1991). 